\section{SAT0592 MORTALITY AND CAUSE OF DEATH IN KOREAN PATIENTS WITH RHEUMATOID ARTHRITIS: BASED ON A LARGE COHORT.}

Y. K. Lee ${ }^{1}$, G. Y. Ahn ${ }^{2}$, J. Lee ${ }^{3}$, J. M. Shin ${ }^{1}$, T. H. Lee ${ }^{1}$, S. C. Bae ${ }^{1,3} .{ }^{1} H_{\text {Hanyang }}$ University Hospital for Rheumatic Diseases, Department of Rheumatology, Seoul, Korea, Rep. of (South Korea); ${ }^{2}$ Korea University Guro Hospital, Division of Rheumatology, Department of Internal Medicine, Seoul, Korea, Rep. of (South Korea); ${ }^{3}$ Hanyang University Institute for Rheumatology Research, Seoul, Korea, Rep. of (South Korea)

Background: Rheumatoid arthritis (RA) is a common chronic inflammatory disease characterized by arthritis of multiple joints. Although the use of corticosteroid and extra-articular complications may lead increased mortality of patients with RA and it have been confirmed by hundreds of studies, the prognosis of RA has improved over the past decades with the introduction of biologics disease-modifying anti-rheumatic drugs and treat-to-target strategy. Along with the increase of overall survival of RA, the needs for re-assessment of actual life expectancy in patients with RA have also been increased.

Objectives: To investigate the cause and the risk of death of Korean patients with RA in a large RA cohort.

Methods: We analyzed patients in Hanyang BAE RA cohort who fulfilled the American College of Rheumatology criteria. A total of 2,355 patients were enrolled from October 2001 to December 2015. Mortality data were derived by linking with data from the Korean National Statistical Office and date and cause death were identified. Standardized Mortality Ratio (SMR) was estimated by dividing the observed deaths by the expected number of deaths of age- and sex- matched general population. Confidence intervals were calculated based on the Poisson distribution.

Table 1. Comparison of demographic and clinical characteristics of analyzed patients

\begin{tabular}{lllll}
\hline & Total & $\begin{array}{l}\text { Died } \\
\text { patients }\end{array}$ & Survivors & P value \\
& $(\mathrm{n}=2,355)$ & $(\mathrm{n}=225)$ & $(\mathrm{n}=2,130)$ & \\
\hline Age at onset & $41.8 \pm 12.8$ & $49.7 \pm 12.7$ & $41.0 \pm 12.5$ & $<0.001$ \\
Age at enrollment & $50.8 \pm 12.3$ & $63.3 \pm 9.4$ & $49.5 \pm 11.8$ & $<0.001$ \\
Male & $265(11.3)$ & $42(18.7)$ & $223(10.5)$ & $<0.001$ \\
Disease duration & $18.1 \pm 10.4$ & $20.7 \pm 10.9$ & $17.8 \pm 10.3$ & $<0.001$ \\
Observation period & $9.0 \pm 4.4$ & $7.0 \pm 3.6$ & $9.2 \pm 4.5$ & $<0.001$ \\
Smoking & & & & $<0.001$ \\
$\quad$ Never smoker & $1,953(83.9)$ & $161(74.9)$ & $1,792(84.8)$ & \\
$\quad \begin{array}{l}\text { Former smoker } \\
\quad \text { Current smoker }\end{array}$ & $212(9.1)$ & $36(16.7)$ & $176(8.3)$ & \\
Comorbidity & $164(7.0)$ & $18(8.4)$ & $146(6.9)$ & \\
$\quad$ Hypertension & $330(14.0)$ & $63(28.0)$ & $267(12.5)$ & $<0.001$ \\
$\quad$ Diabetes mellitus & $106(4.5)$ & $24(10.7)$ & $82(3.9)$ & $<0.001$ \\
\hline
\end{tabular}

*Values are number $(\%)$ or mean \pm standard deviation. ${ }^{* *}$ Missing data were excluded from analysis.

Results: Over the observation period, 225 deaths were reported. The age at enrollment was $50.8 \pm 12.3$ years and disease duration was $18.1 \pm 10.4$ years. The most common cause of death was malignancy ( 40 cases) followed by respiratory disease (38 cases), cardiovascular disease (32 cases) and musculoskeletal disease (21 cases). Total SMR was increased [1.7, 95\% Cl 1.5-2.0] but age- and sex- adjusted SMR was not increased [SMR 1.0, $(95 \% \mathrm{Cl} 0.9-1.1)]$. When we classify patients by age conduct subgroup analysis, sex-adjusted SMR was also similar with that of general population by all group: the adjusted SMR in patients aged 15-39, aged 40-59 and aged over 60 were 0.7 (observed death 1, expected death 1.5, 95\% $\mathrm{Cl} 0-2.0$ ), 0.9 (observed death 29, expected death 32 95\% Cl 0.6-1.3), and 0.9 (observed death 195, expected death $221.0,95 \% \mathrm{Cl} 0.8-1.0$ ), respectively. Compared with survivors, patients who died were more likely to be male $(18.7 \%$ vs. $10.5 \%, p<0.001)$ and they had an older age of onset of RA ( 49.7 years vs. 41.0 years, $p<0.001$ ). Died patients were more likely to be current smoker or ever smoker $(25.1 \%$ vs. $15.2 \%$, $\mathrm{p}<0.001)$ and accompanied by more comorbidities including hypertension $(28.0 \%$ vs. $12.5 \%, p<0.001)$ and diabetes mellitus $(10.7 \%$ vs. $3.9 \%, p<0.001)$.

Conclusion: The overall age- and sex- matched SMR of patients with RA was similar with that of general population. However, compared with survivors, patients who died were more likely to be male, diagnosed with RA at older age, more likely to be smoker, and they have more hypertension and diabetes mellitus. Therefore, attention should be paid not only to RA itself but also managing comorbidities to improve the survival of patients with RA

Disclosure of Interests: None declared

DOI: 10.1136/annrheumdis-2020-eular.3434

\section{SAT0593 \\ ANA TESTING IN THE (VERY) ELDERLY: EXPECTATION VERSUS REALITY}

M. Luis $^{1,2}$, A. Carmo ${ }^{3}$, R. Cunha ${ }^{3}$, J. A. P. Da Silva ${ }^{1,2,4}$, T. Santiago ${ }^{1,2} .{ }^{1}$ Centro Hospitalar e Universitário de Coimbra, Rheumatology, Coimbra, Portugal; ${ }^{2}$ Faculty of Medicine, University of Coimbra, Coimbra, Portugal; ${ }^{3}$ Centro Hospitalar e Universitário de Coimbra, Clinical Pathology, Coimbra, Portugal; ${ }^{4}$ Coimbra Institute for Clinical and Biomedical Research (iCBR), Coimbra, Portugal

Background: Antinuclear antibodies (ANA) are frequently used as a screening tool for systemic autoimmune rheumatic diseases (SARD), although they are also present in $10-15 \%$ of the adult healthy population. SARD have their peak incidence in the young/ middle-aged adult. As age progresses, the incidence of SARD decreases while the prevalence of ANA tends to increase, with some series reporting up to $30 \%$ prevalence in older ages ${ }^{1}$.

Objectives: To determine the clinical significance and utility of ANA testing in a population over 85 years of age.

Methods: We conducted a retrospective study of patients over the age of 85 who underwent ANA testing due to a SARD suspicion at our hospital autoimmunity laboratory, from 2011 to 2018. Justification for ANA request was collected from patient's clinical records. Patients with pre-established diagnosis of SARD and patients with no justification given for ANA request were excluded from the analysis. ANA titer (positive $\geq 1: 160$ ) and cellular staining patterns were assessed by indirect immunofluorescence (Hep-2 cells).

Results: Ages ranged from 85 to 98 years, with $58.8 \%$ being females. The prevalence of ANA in this population was $61.5 \%$, mostly in lower titers (1:160 in $45.0 \%, 1: 320$ in $31.9 \%, 1: 640$ in $20.3 \%$ and $1: 1280$ in $2.7 \%$ ). Dense fine speckled pattern was by far the most common cellular staining pattern $(79.1 \%)$. A suspicion of SARD was the reported reason for ANA testing in 34,5\% $(n=296)$ of the 854 patients submitted to this test. The main clinical clues justifying SARD suspicion were: arthralgia/arthritis (11.9\%), thrombocytopenia $(10.0 \%)$, pancytopenia $(10.0 \%)$, spotless fever $(8.2 \%)$, interstitia lung disease (4.8\%), pleural (6.1\%) and pericardial (4.1\%) effusion. Over a median follow-up of 1.0 year, 10 patients $(3.4 \%)$ were diagnosed with a SARD, only one being an ANA-related disease: 5 cases of polymyalgia rheumatica, 2 cases of rheumatoid arthritis, 1 case of giant cell arteritis, 1 case of Sjogren syndrome and 1 case of sarcoidosis. In $60 \%$ of patients with a confirmed SARD, the main reason for suspicion was the presence of arthralgia/ arthritis. Positive ANA testing showed a $90.0 \%$ sensitivity and a $39.6 \%$ specificity for SARD. This translates into a positive predictive value of $5.0 \%$. Conclusion: ANA are highly prevalent in elderly patients under SARD suspicion, while the incidence of SARD is very low, which explains the low positive predic tive value of ANA testing. Interestingly, only one among the ten cases of SARD confirmed was indeed an ANA-related disease (Sjogren syndrome).

References:

[1] Selmi C, Ceribelli A, Generali E, et al. Serum antinuclear and extractable nuclear antigen antibody prevalence and associated morbidity and mortality in the general population over 15 years. Autoimmun Rev. 2016;15(2):162166. doi:10.1016/j.autrev.2015.10.007

Disclosure of Interests: Mariana Luis: None declared, Anália Carmo: None declared, Rosário Cunha: None declared, José Antonio P. da Silva Grant/ research support from: Pfizer, Abbvie, Consultant of: Pfizer, AbbVie, Roche, Lilly, Novartis, Tânia Santiago: None declared DOI: 10.1136/annrheumdis-2020-eular.2359

\section{SAT0594 LONG-TERM MORBIDITY FOLLOWING IGA VASCULITIS IN CHILDHOOD}

J. “ Nossent ${ }^{1,2}$, W. Raymond ${ }^{1}$, M. Ognjenivic ${ }^{1}$, H. Keen ${ }^{1,3}$, D. Preen ${ }^{4}$

C. Inderjeeth ${ }^{1,2}{ }^{1}$ The University of Western Australia, Rheumatology section, School of Medicine, Crawley, Australia; ${ }^{2}$ Sir Charles Gairdner Hospital, Rheumatology, Nedlands, Australia; ${ }^{3}$ Fiona Stanley Hospital, Rheumatology, Murdoch, Australia; ${ }^{4}$ The University of Western Australia, School of Population and Global Health, Crawley, Australia

Background: $\lg A$ vasculitis ( $\lg A \mathrm{~V})$ in children is considered a mostly self-limiting disease. However, patients may require aggressive initial treatment, are prone to disease relapses and conceivably have a sustained abnormality in mucosal and /or circulating $\lg$ A responsiveness, that can predispose to the development of other conditions. Objectives: To determine whether childhood IgAV predisposes to comorbidity later in life.

Methods: Observational cohort study examining rates of hospitalization, ED visits, procedures and accrual of comorbidity (by Charlson comorbidity index; $\mathrm{CCl}$ ) comparing $494 \mathrm{IgAV}$ patients $<20$ years at diagnosis with 1385 non-exposed matched controls over a 20-year period. Maximum likelihood estimates were used to obtain Odds (OR) and Rate ratios per 1000 person-years (RR).

Results: Hospitalization was increased proportionally (73.5 vs $51.5 \%$ ) and by rate $(21.7$ vs 18.9 ; rate ratio 1.15$)$ (both $p<0.01$ ) for IgAV patients, who underwent more diagnostic and medical procedures whereas controls had higher rates of surgical interventions. IgAV patients had an higher overall ED attendance (25 vs $16 \%$ ) and visit rate (10.8 vs 8.43 , RR 1.29 ) (each $p<0.01)$ ) and accrued more often peptic ulcer and renal disease and developed severe comorbidity $(\mathrm{CCl} \geq 3)$ at a higher rate (OR 2.9, $95 \% \mathrm{Cl} 0.79-11.6$ ) than controls.

Conclusion: A diagnosis of $\operatorname{IgAV}$ in childhood associates with increased risk and rate of subsequent hospital admission, ED attendance and severe comorbidity. 
The occurrence of childhood IgAV thus signifies the presence of a sustained predisposition to illness.

Acknowledgments: Supported by an unrestricted grant from the Arthritis Foundation of Western Australia

Disclosure of Interests: Johannes ("Hans") Nossent Speakers bureau: Janssen, warren raymond: None declared, milica ognjenivic: None declared, Helen Keen Speakers bureau: Pfizer Austrlaia, Abbvie Australia, David Preen: None declared, Charles Inderjeeth Grant/research support from: UCB Australia, Speakers bureau: Eli Lilly

DOI: 10.1136/annrheumdis-2020-eular.639

\begin{tabular}{l|l}
\hline SAT0595 & INTERSTITIAL LUNG DISEASE IN SOUTH AFRICAN \\
& ADULTS PATIENTS WITH AUTOIMMUNE RHEUMATIC \\
& DISEASES
\end{tabular}

E. Palalane $^{1}$, D. Alpizar-Rodriguez ${ }^{2}$, S. Botha3, Q. M. S. H. Said-Hartley ${ }^{4}$, G. Calligaro ${ }^{5}$, B. Hodkinson ${ }^{1}{ }^{1}$ University of Cape Town - Groote Schuur Hospital, Rheumatology, Cape Town, South Africa; ${ }^{2}$ University Hospitals of Geneve, Rheumatology, Geneve, Switzerland; ${ }^{1}$ University of Cape Town - Groote Schuur Hospital, Rheumatology, Cape Town, South Africa; ${ }^{4}$ University of Cape Town Groote Schuur Hospital, Radiology, Cape Town, South Africa; ${ }^{5}$ University of Cape Town - Groote Schuur Hospital, Pulmonology, Cape Town, South Africa

Background: Interstitial lung disease (ILD) is prevalent in patients with autoimmune rheumatic diseases (ARD), leads to significant morbidity and mortality, and is poorly characterized in South Africa.

Objectives: To describe the clinical, serological and radiological features of ILD associated with ARD in a tertiary referral hospital.

Methods: A cross-sectional study of outpatients attending the rheumatology and respiratory clinics of Groote Schuur Hospital between October 2018 and September 2019. Clinical, serological and radiological features were documented. We compared features of 3 groups of patients: rheumatoid arthritis (RA), systemic sclerosis (SSc) and "Other" autoimmune rheumatic diseases (OARD) which included Idiopathic Inflammatory Myopathies, Mixed Connective Tissue Disease, Systemic Lupus Erythematous, ANCA-associated vasculitis, Sjogren's Syndrome and overlap syndromes. Factors associated with Usual Interstitial Pneumonia (UIP) were sought by univariate and multivariate analysis. P-values $\leq 0.05$ were considered statistically significant. Analyses was performed with STATA 14.0 (Stata Corp LP, USA).

Results: Of 124 patients, $29.8 \%$ had RA, 25,8 \% SSc and $44.4 \%$ OARD. Most patients were female (86.3\%), of mixed racial ancestry $(75.0 \%)$, and the median (IQR) age was 55 (46-66). Over one-third were smokers, $22.6 \%$ had emphysema, and one third had previous pulmonary tuberculosis (PTB) infection. Smoking, emphysema, and previous PTB were higher in RA group but the difference was not statistically significant. All SSc patients and more than two-thirds of RA and OARD patients had gastroesophageal reflux disease (GORD).

Similar to reports elsewhere, Nonspecific interstitial pneumonia (NSIP) was the commonest ILD (63.7\%), followed by UIP $(26.6 \%)$ and other patterns $(9.7 \%)$. Contrary to other reports, we found similar frequencies of NSIP and UIP patterns in patients with RA. RA patients were significantly older (median (IQR)) age at ILD onset 62 (55-68) years, compared to SSc (49 (38-56) and OARD (42 (33-56) $(\mathrm{p}<$ $0.001)$. The percentage of predicted Forced Vital Capacity (FVC) were significantly worse in SSc and OARD groups and DLCO in OARD. RA diagnosis (OR 3.8, 95\% $\mathrm{Cl} 1.5-9.5$ ), older age (OR 1.1, 95\% Cl 1.0-1.1), COPD (OR 3.2, 95\% Cl 1.4-8.0), longer ARD-ILD interval, higher FVC (OR 1.0, 95\% Cl 1.0-1.1) and previous Methotrexate (MTX) use (OR 2.6, 95\% Cl 1.1-6.0) were significantly associated with UIP. Multivariable analysis showed that only COPD and previous MTX use was associated with UIP (OR $2.8(95 \% \mathrm{Cl} 1.0-8.0)$ and 1.0 (95\% Cl $1.0-1.0)$ respectively). Regarding MTX exposure, $37.1 \%$ of patients were prescribed MTX before ILD diagnosis, and $33.9 \%$ continued, started or restarted after ILD diagnosis. No case of acute pneumonitis was documented.

Conclusion: ILD was most commonly diagnosed in RA and SSc, with NSIP seen most frequently overall. RA patients presented better Pulmonary function tests despite higher frequency of UIP. The use of MTX seems to not be associated with the development of acute pneumonitis in patients with ILD

References:

[1] Wallace, B., D. Vummidi, and D. Khanna, Management of connective tissue diseases associated interstitial lung disease: a review of the published literature. Current Opinion in Rheumatology, 2016. 28(3): p. 236-245.

[2] Dellaripa, P.F., Interstitial lung disease in the connective tissue diseases; a paradigm shift in diagnosis and treatment. Clinical Immunology, 2018. 186: p. 71-73.

Disclosure of Interests: None declared

DOI: 10.1136/annrheumdis-2020-eular.4888

\section{SAT0596 \\ ASSOCIATIONS BETWEEN CIRCULATING LIPID MEDIATORS AND INCIDENT INFLAMMATORY ARTHRITIS IN AN ANTI-CITRULLINATED PROTEIN ANTIBODY POSITIVE POPULATION}

K. Polinski ${ }^{1}$, E. Bemis ${ }^{1}$, K. Demoruelle ${ }^{2}$, J. Seifert ${ }^{2}$, T. Crume ${ }^{1}$, F. Yang ${ }^{3}$, W. Robinson ${ }^{4}$, M. Clare-Salzler ${ }^{5}$, K. Deane ${ }^{2}$, M. Holers ${ }^{2}$, J. Norris ${ }^{1}{ }^{1}{ }^{1}$ University of Colorado Anschutz Medical Campus (CU Anschutz), Epidemiology, Aurora, United States of America; ${ }^{2}$ University of Colorado Anschutz Medical Campus (CU Anschutz), Aurora, United States of America; ${ }^{3}$ University of Colorado Anschutz Medical Campus (CU Anschutz), Biostatistics and Informatics, Aurora, United States of America; ${ }^{4}$ Stanford, Stanford, United States of America; ${ }^{5}$ University of Florida, Gainesville, United States of America

Background: Lipid mediators are endogenously derived from the metabolism of omega-3 and omega-6 polyunsaturated fatty acids (PUFAs) and have importan roles in promoting and resolving inflammation in the body (1). Epidemiologica studies have shown higher omega-3 PUFA status to be associated with a lower risk of both autoimmunity and progression to inflammatory arthritis $(I A)(2,3)$. Objectives: To determine the association of lipid mediators with progression from rheumatoid arthritis (RA)-related autoimmunity to inflammatory arthritis (IA). Methods: We conducted a prospective cohort study using data from the Studies of the Etiologies of Rheumatoid Arthritis (SERA). SERA enrolled first-degree relatives (FDRs) of individuals with RA (FDR cohort) and individuals who screened positive for RA-related autoantibodies at health fairs (screened cohort). We followed 133 antiCCP3.1 positive participants, of which 29 developed IA (22 classified as RA by 2010 ACR/EULAR criteria). We quantified lipid mediators from stored plasma samples via liquid chromatography tandem mass spectrometry methods validated against the collection and storage methods used in the study. A priori, we selected 5S-HETE, 15S-HETE and 17S-HDHA because they are precursors to leukotrienes, Lipoxin A4 and Resolvin D series lipid mediators, respectively. We fit Cox proportional hazard models for each lipid mediator as a time-varying covariate. For lipid mediators significantly associated with progression to IA we then examined IL-1 $\beta$, IL-6, IL-8 and TNF-a (Bio-Plex Pro $^{T M}$ assay) as potential mediators of this relationship.

Results: Higher plasma 5S-HETE levels were associated with an increased risk of incident IA after adjusting for age at baseline, cohort (FDR or screened), and shared epitope (SE) status (Table 1). The models examining 15S-HETE and $17 \mathrm{~S}-\mathrm{HDHA}$ had the same trend but did not reach statistical significance. We did not find evidence that the association between 5S-HETE and IA risk was mediated by the tested pro-inflammatory cytokines, suggesting a direct role for this lipid mediator in conversion to IA.

Table 1. Hazard ratios and $95 \%$ confidence intervals of lipid mediator concentrations associated with IA, $n=29$ IA cases

\begin{tabular}{lcc}
\hline Lipid mediator & Crude & Adjusted $^{\mathrm{b}}$ \\
\hline $5 \mathrm{~S}-\mathrm{HETE}$ & $2.10(1.12,3.92)$ & $2.41(1.43,4.07)$ \\
$15 \mathrm{~S}-\mathrm{HETE}$ & $1.61(0.88,2.93)$ & $1.52(0.87,2.65)$ \\
$17-\mathrm{HDHA}^{\mathrm{a}}$ & $1.59(0.68,3.74)$ & $1.61(0.72,3.56)$ \\
\hline
\end{tabular}

a dichotomized as <limit of detection (reference) or detected

${ }^{\mathrm{b}}$ Adjusted for SE, age at baseline and cohort

Conclusion: In a prospective cohort of anti-CCP positive individuals, higher circulating levels of 5S-HETE, an important precursor to pro-inflammatory leukotrienes, was associated with subsequent IA. Our findings highlight the potential pathologic and prognostic significance of these PUFA metabolites in inflammatory processes in pre-RA populations.

References:

[1] Serhan CN. Pro-resolving lipid mediators are leads for resolution physiology. Nature. 2014;510(7503):92-101.

[2]Gan RW, Bemis EA, Demoruelle MK, Striebich CC, Brake S, Feser ML, et al. The association between omega-3 fatty acid biomarkers and inflammatory arthritis in an anti-citrullinated protein antibody positive population. Rheumatology. 2017.

[3] Gan RW, Young KA, Zerbe GO, Demoruelle MK, Weisman MH, Buckner JH, et al. Lower omega-3 fatty acids are associated with the presence of anti-cyclic citrullinated peptide autoantibodies in a population at risk for future rheumatoid arthritis: a nested case-control study. Rheumatology. 2016;55(2):367-76.

Disclosure of Interests: Kristen Polinski: None declared, Elizabeth Bemis: None declared, Kristen Demoruelle Grant/research support from: Pfizer, Jennife Seifert: None declared, Tessa Crume: None declared, Fan Yang: None declared William Robinson: None declared, Michael Clare-Salzler: None declared Kevin Deane Grant/research support from: Janssen, Consultant of: Inova ThermoFisher, Janseen, BMS and Microdrop, Michael Holers Shareholder of AdMIRx, Grant/research support from: AdMIRx, Pfizer, Janssen R\&D, Consultant of: AdMIRx, Janssen R\&D, Celgene, Bristol-Myers Squibb, Jill Norris Grant research support from: Janssen R\&D, Pfizer, Consultant of: Celgene, BMS DOI: 10.1136/annrheumdis-2020-eular. 1884

\section{SAT0597 EARLY DAMAGE AS MEASURED BY SLICC/ ACR DAMAGE INDEX IS A PREDICTOR OF HOSPITALIZATION IN SYSTEMIC LUPUS ERYTHEMATOSUS (SLE)}

L. Raman ${ }^{1}$, F. Yahya ${ }^{1}$, C. M. Ng${ }^{2}$, S. Sockalingam ${ }^{1}$, R. J. B. Raja Mohamed ${ }^{1}$. ${ }^{1}$ University of Malaya, Division of Rheumatology, Department and Faculty 